\title{
NOISE-INDUCED CHAOS AND BACKWARD STOCHASTIC BIFURCATIONS IN THE LORENZ MODEL
}

\author{
IRINA BASHKIRTSEVA* and LEV RYASHKO ${ }^{\dagger}$ \\ Ural Federal University, Lenina, 51, \\ 620083, Ekaterinburg, Russia \\ *irina.bashkirtseva@usu.ru \\ †lev.ryashko@usu.ru \\ PAVEL STIKHIN \\ KCS.net \\ St. Gallen, Switzerland
}

Received August 20, 2012; Revised February 18, 2013

\begin{abstract}
We study the phenomena of stochastic D- and P-bifurcations of randomly forced limit cycles for the Lorenz model. As noise intensity increases, regular multiple limit cycles of this model in a period-doubling bifurcations zone are deformed to be stochastic attractors that look chaotic (D-bifurcation) and their multiplicity is reduced (P-bifurcation). In this paper for the comparative investigation of these bifurcations, the analysis of Lyapunov exponents and stochastic sensitivity function technique are used. A probabilistic mechanism of backward stochastic bifurcations for cycles of high multiplicity is analyzed in detail. We show that for a limit cycle with multiplicity two and higher, a threshold value of the noise intensity which marks the onset of chaos agrees with the first backward stochastic bifurcation.
\end{abstract}

Keywords: Noise-induced chaos; backward stochastic bifurcation; stochastic sensitivity function; Lorenz model.

\section{Introduction}

One of the most attractive problems in nonlinear science is the interplay between chaos and noise. The effect of noise on period-doubling transition to chaos was studied in [Crutchfield et al., 1981; Crutchfield et al., 1982] where for scaling analysis of Lyapunov exponents, a renormalization group approach was used. The influence of noise on the periodic attractors of the logistic model was investigated in [Mayer-Kress \& Haken, 1981] on the base of the analysis of stationary distributions of corresponding stochastic attractors. Main approaches suggested in these pioneer works were widely developed by many researchers [Landa \& McClintock, 2000; Anishchenko et al., 2007].
In a modern theory of nonlinear stochastic dynamics, two types of stochastic bifurcations are considered [Arnold, 1998]. First, so-called D-bifurcation occurs when dynamical properties of the system are qualitatively changed. Usually, a study of D-bifurcations is based on the analysis of Lyapunov exponents. A noise-induced transition from order to chaos is one of the most attractive examples of D-bifurcations. Negative or zero Lyapunov exponent of noise-free system becomes positive when noise is turned on and its amplitude exceeds some threshold value. In the last decade, a scaling analysis of the Lyapunov exponents for various continuous-time dynamical systems with noise-induced chaotic dynamics has been 
a subject of extensive analysis [Liu et al., 2002; Lai et al., 2003; Liu \& Ma, 2005; Tung et al., 2008; Tel \& Lai, 2010; Pradas et al., 2011].

Another type of bifurcation, so-called P-bifurcation, is connected with a qualitative change of the form of probabilistic distribution of random states in stochastic attractor. P-bifurcations in one- and two-dimensional systems are currently the object of considerable research [Horsthemke \& Lefever, 1984; Arnold et al., 1996, 1999; Rasmussen, 2007; Anishchenko et al., 2007; Bashkirtseva et al., 2009]. P-bifurcations in 3D-systems have just begun to be studied [Bashkirtseva et al., 2010b]. D- and Pbifurcation theory is actively applied in the analysis of various noise-induced effects (see e.g. a study of the noisy neural network in [Huang et al., 2011]).

In the present paper, we study the influence of noise on limit cycles of 3D-systems. Our interest is focused on the noise-induced chaotization of regular periodic oscillations and P-bifurcations observed in a period-doubling zone. As a basic example, we use the familiar Lorenz model which exhibits such period-doubling bifurcations with cycles of high multiplicity [Sparrow, 1982].

The stochastically forced Lorenz model was investigated by direct simulation and analytically by many researchers (see e.g. [Dorfle \& Graham, 1983; Ito, 1984; Keizer et al., 1993; Anishchenko et al., 2001]). An influence of noise on periodic attractors of the Lorenz model was studied in [Fedchenia et al., 1992]. A phenomenon of noise-induced chaos in the Lorenz model was considered in a zone of coexisting stable equilibria [Gao et al., 2002; Tung et al., 2008] and in a periodic window [Liu et al., 2002].

A fundamental dynamic mechanism responsible for the noise-induced transition from order to chaos was found qualitatively: under random disturbances, the flow of trajectories of the forced system can leave the basins of attraction and intersect zones of divergency. Now, for quantitative analysis of this mechanism, it is necessary to develop constructive methods by taking into account the probabilistic and geometrical peculiarities of the studied systems. The widely used technique of Lyapunov exponents allows only to register a noise-induced chaos.

In the present paper, we associate onset of noise-induced chaos with qualitative changes in probability distribution (P-bifurcation) and propose a constructive method for chaos prediction.
Our method is based on stochastic sensitivity functions (SSF) technique. SSF technique was successfully used for the analysis of noise-induced transitions between two separate coexisting limit cycles of the Lorenz system [Bashkirtseva \& Ryashko, 2009]. In [Bashkirtseva et al., 2012], this technique was applied in the analysis of noise-induced chaos generated by random hopping between two separated but close portions of a single cycle. In this paper, we consider another, but more general geometrical case. We focus our interest on the cycles in a period-doubling bifurcations zone. One of the aims of the presented paper is to interconnect the geometrical dynamical mechanism of noise-induced chaotization for multiple cycles of Lorenz system with a special kind of P-bifurcations, namely backward stochastic bifurcations (BSB).

In the study of P-bifurcations, a constructive description of the probability density function of the investigated stochastic attractor is required. This function is governed by the KolmogorovFokker-Planck (KFP) equation. The analysis of KFP equation for stochastic 3D-cycles considered here is a very difficult problem technically. In this case, asymptotics and approximations based on quasipotential and stochastic sensitivity functions (SSF) have been elaborated [Freidlin \& Wentzell, 1984; Mil'shtein \& Ryashko, 1995; Bashkirtseva \& Ryashko, 2004]. SSF technique was successfully applied to the analysis of stochastic cycles for Chua's circuit [Ryagin \& Ryashko, 2004] and Chen system [Bashkirtseva et al., 2010a], and for the spatial probabilistic description of 3D stochastic cycles in a form of confidence tori [Ryashko et al., 2009].

For multiple cycles, noise induces a special type of P-bufurcations, so-called backward stochastic bifurcations (BSB): as the noise intensity increases, peaks of the probability density function merge and multiplicity of cycle is reduced. These bifurcations have been initially discussed in [Mayer-Kress \& Haken, 1981] for discrete-time logistic model. Bifurcation analysis of stochastic multiple cycles for 3D continuous-time systems via direct numerical simulation is very costly for long-time direct numerical simulations of random trajectories. As the multiplicity of studied cycle increases, the expenses rise extremely.

For the analysis of BSB in 3D continuoustime systems, a constructive approach using SSF technique has been proposed in [Bashkirtseva et al., 2010b] where only first backward stochastic 
bifurcations of randomly forced Rössler system have been studied.

In the present paper, SSF technique is extended for the analysis of the whole chain of BSB $2^{k} \rightarrow$ $2^{k-1} \rightarrow \cdots \rightarrow 2^{0}=1$ for stochastic cycles of higher multiplicity. It is shown that the elaborate technique is universal and constructive for the investigation of stochastic 3D-cycles with various multiplicity in the period-doubling bifurcation zone.

In Sec. 2, stochastic D-bifurcations for the Lorenz model are investigated. Here, a phenomenon of noise-induced transition from order to chaos is studied for limit cycles of various multiplicity via an analysis of largest Lyapunov exponents. It is shown that for a limit cycle with multiplicity two and higher, there exists a threshold value of noise intensity which marks the onset of chaos. This threshold essentially depends on the multiplicity of the forced cycle.

In Sec. 3, P-bifurcations of stochastic cycles are investigated. A phenomenon of backward stochastic bifurcations of stochastic cycles of various multiplicity is studied via a stochastic sensitivity function technique. Details of this general technique are shortly reviewed in the Appendix. For multiple 2-, 4-,8-,16-cycles, sets of threshold values for whole chains of BSB are found. It is discussed how the noise-induced transition from order to chaos relates with the backward stochastic bifurcations.

\section{Noise-Induced Chaotization of Regular Periodic Attractors of Lorenz System}

Consider the stochastically perturbed Lorenz system:

$$
\begin{aligned}
& \dot{x}=\sigma(-x+y)+\varepsilon \dot{w}_{1} \\
& \dot{y}=r x-y-x z+\varepsilon \dot{w}_{2} \quad \sigma=10, \quad b=\frac{8}{3} \\
& \dot{z}=-b z+x y+\varepsilon \dot{w}_{3} .
\end{aligned}
$$

Here $w_{i}(t)(i=1,2,3)$ are independent standard Wiener processes with Gaussian increments, $E\left(w_{i}(t)-w_{i}(s)\right)=0, E\left(w_{i}(t)-w_{i}(s)\right)^{2}=|t-s|$. Parameter $\varepsilon$ is a value of the noise intensity.

For $\varepsilon=0$, on the interval $205 \leq r \leq 235$, the deterministic Lorenz system demonstrates both regular and chaotic dynamics. In Fig. 1, a bifurcation diagram is given. Here, we plot $z$-coordinates of the intersection points of deterministic attractors with a secant plane $y=0$ for $x<0$.

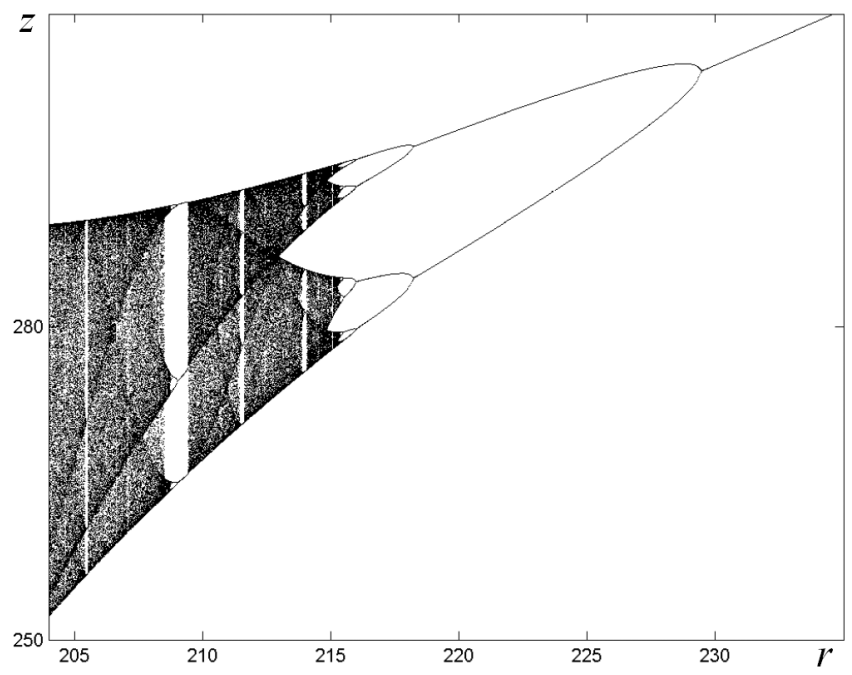

Fig. 1. Bifurcation diagram of the deterministic Lorenz system.

In this paper, we focus on limit cycles in the well-known [Sparrow, 1982] period-doubling bifurcations zone $I=\bigcup_{k=0}^{\infty} I_{k}$. Here $I_{k}$ is an interval of structural stability, where a stable $2^{k}$-cycle is observed: $I_{0}=(229,235), \quad I_{1}=(218.22,229)$, $I_{2}=(215.88,218.22), I_{3}=(215.49,215.88), I_{4}=$ $(215.39,215.49), \ldots$ In each interval $I_{k}$, we fix a parameter value $r_{k}: r_{0}=230$ (1-cycle), $r_{1}=224.5$ (2-cycle), $r_{2}=217.2$ (4-cycle), $r_{3}=215.75$ (8cycle), $r_{4}=215.45$ (16-cycle). These $r_{k}$ are close to the values which correspond to the most stable limit cycles (so-called "supercycles"). In Fig. 2, deterministic limit cycles of Lorenz systems for these values of the parameter $r$ are plotted.

Under random disturbances, the trajectories of the stochastically forced Lorenz system leave the deterministic attractor and form some stochastic attractor around it. In Fig. 3, a stochastic 1-cycle for $r_{0}=230, \varepsilon=0.5$ is plotted.

The influence of noise on the whole perioddoubling interval can be illustrated by a stochastic bifurcation diagram. In Fig. 4, the diagrams for three different values of the noise intensity $\varepsilon$ are shown. Here, a thin structure of the unforced bifurcation diagram is washed out by noise. Rigorous theoretical analysis of a probabilistic mechanism for this washing-out will be presented in Sec. 3 in a framework of backward P-bifurcations theory developed here.

As one can see, random disturbances essentially deform periodic attractors of Lorenz system. From the beginning, we examine how dynamical properties of stochastic cycles of the Lorenz system 
(a)

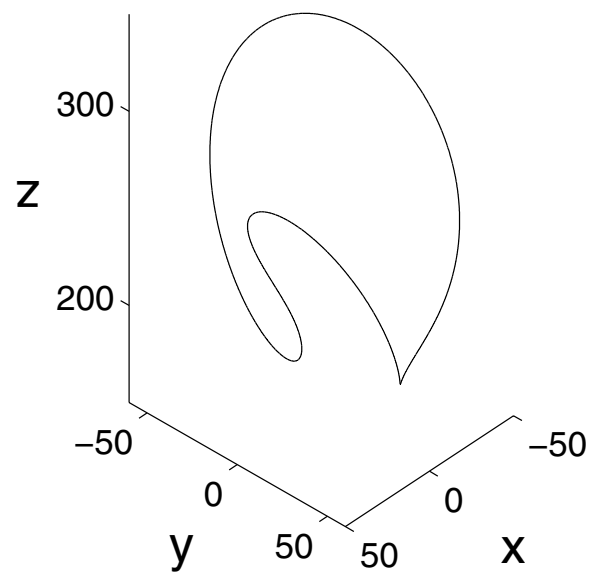

(c)

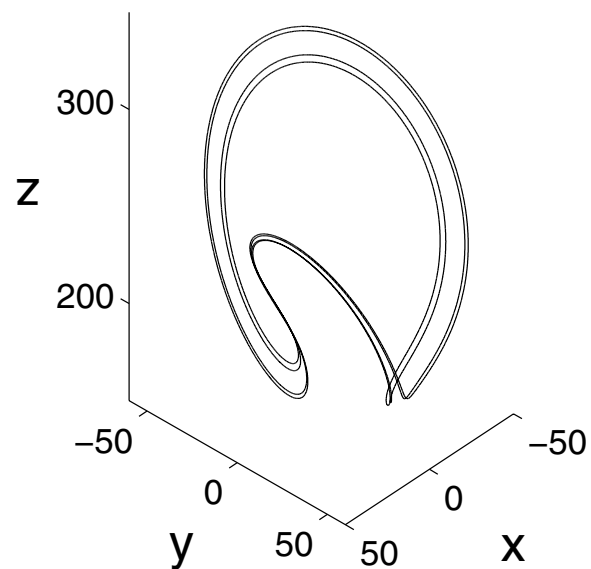

(b)

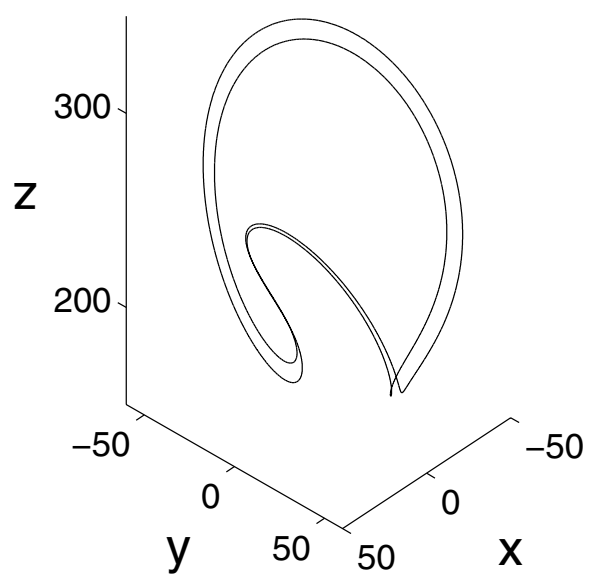

(d)

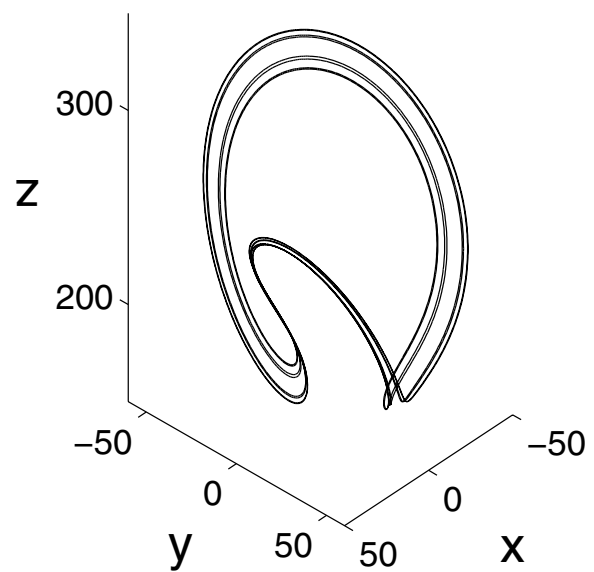

Fig. 2. Deterministic cycles of Lorenz system: (a) $r_{0}=230$ (1-cycle), (b) $r_{1}=224.5$ (2-cycle), (c) $r_{2}=217.2$ (4-cycle) and (d) $r_{3}=215.75$ (8-cycle).

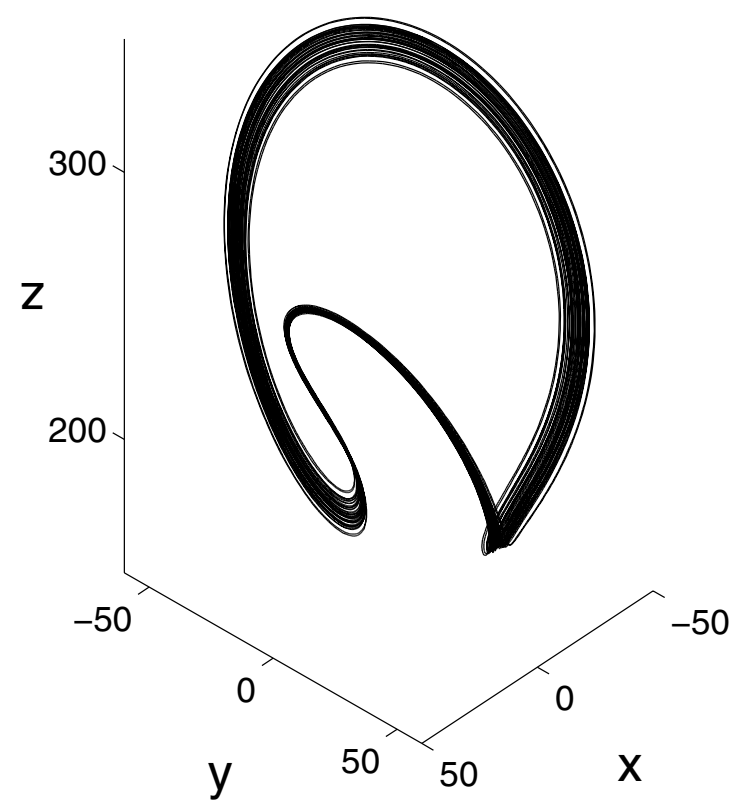

Fig. 3. Stochastic 1-cycle of Lorenz system for $r_{0}=230$ and $\varepsilon=0.5$. depend on the noise intensity $\varepsilon$ and cycle's multiplicity. The largest Lyapunov exponent is a standard quantitative measure of the nonlinear system dynamics as a whole. In Fig. 5, plots of largest Lyapunov exponents $\Lambda(\varepsilon)$ are presented for parameter values $r_{0}, r_{1}, r_{2}, r_{3}$ corresponding to cycles of various multiplicities. Here for all $r_{i}$, we have $\Lambda(0)=0$, which is theoretically clear. For $\varepsilon>0$, the Lyapunov exponent essentially depends on the multiplicity of considered limit cycles. For stochastic 1-cycle (circles), the function $\Lambda$ has values close to zero. So, the stochastic 1-cycle can be characterized here as a regular attractor. For stochastic 2 -cycle (triangles), beyond some threshold $\varepsilon^{*} \approx 0.6$, the values of $\Lambda(\varepsilon)$ are definitely positive. Moreover, the function $\Lambda(\varepsilon)$ monotonically grows. This transition to positive values of the Lyapunov exponent can be interpreted as a D-bifurcation [Arnold, 1998] which marks the onset of the noise-induced chaotization. For stochastic 4- and 8-cycles, these 


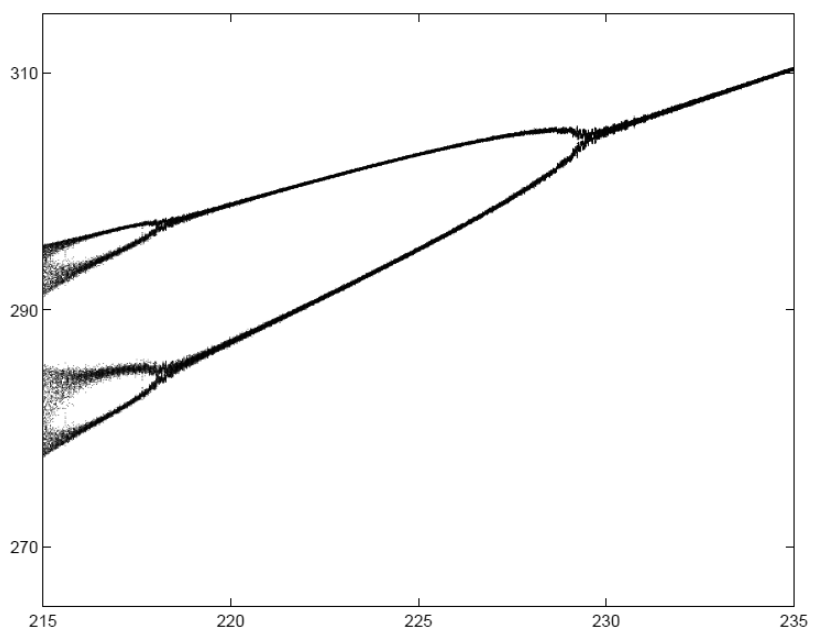

(a)

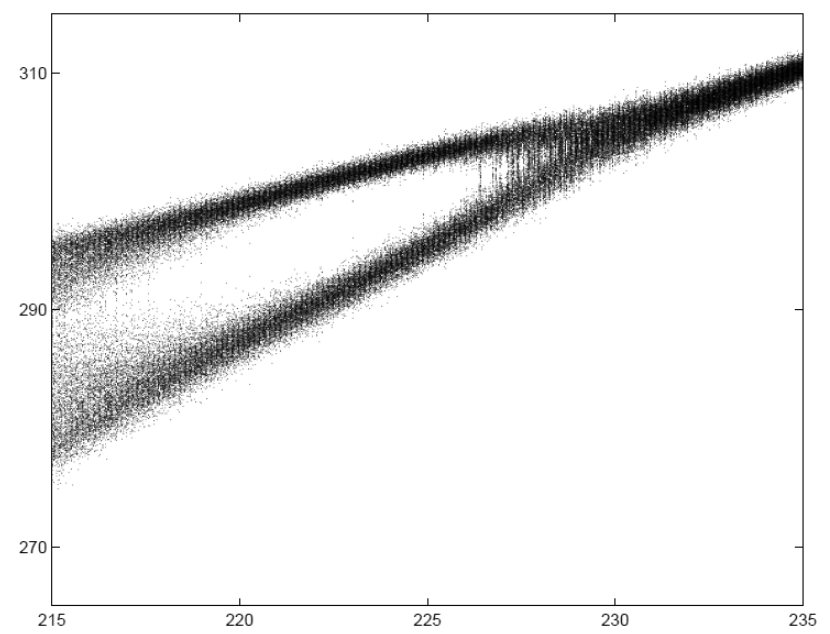

(b)

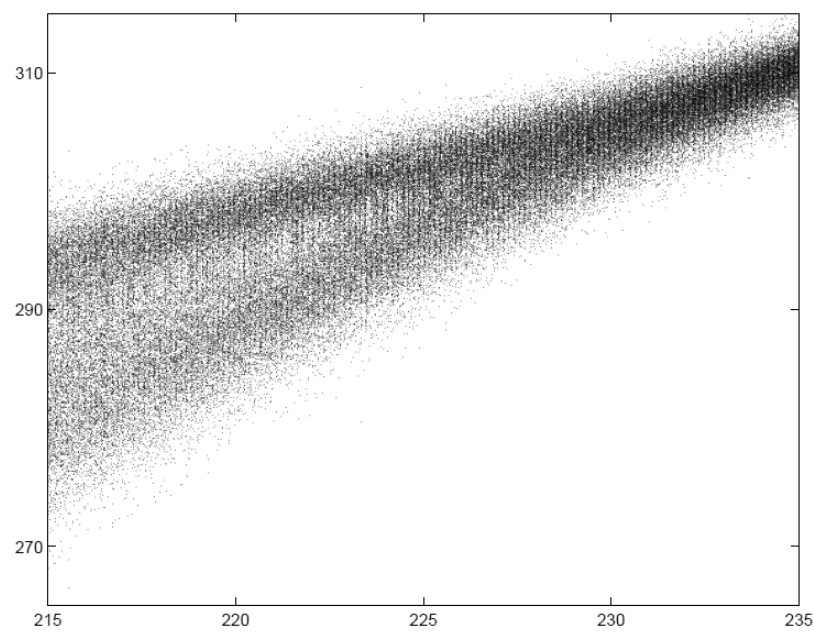

(c)

Fig. 4. Bifurcation diagram of the stochastic Lorenz system: (a) $\varepsilon=0.01$, (b) $\varepsilon=0.1$ and (c) $\varepsilon=0.5$.

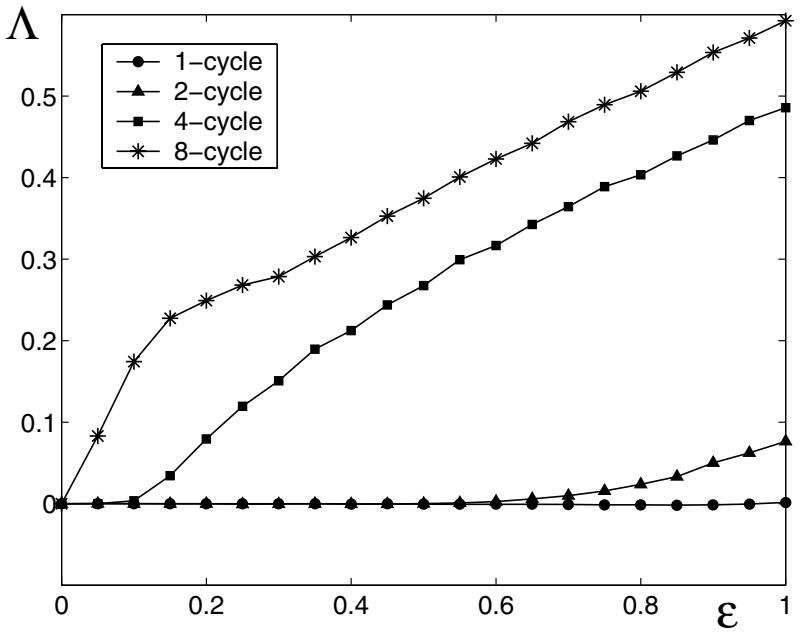

Fig. 5. Largest Lyapunov exponent $\Lambda(\varepsilon)$ for 1-cycle (circles), 2-cycle (triangles), 4-cycle (squares), and 8-cycle (asterisks). transitions to chaos are observed for lesser noise levels. So, the threshold noise intensity corresponding to the transition from order to chaos, essentially depends on the multiplicity of cycles. An understanding of the underlying theoretical reason of such dependence can be acquired via a comparison of the stochastic sensitivity for the cycles of different multiplicities.

It is known that stochastic sensitivity of cycles increases in the chain of period-doubling bifurcations: the more multiplicity, the higher sensitivity. Under the transition from order to chaos, the stochastic sensitivity of limit cycles tends to infinity. It has been studied for cycles of Chua's circuit [Ryagin \& Ryashko, 2004] and Chen system [Bashkirtseva et al., 2010a] by the SSF technique. In Sec. 3, we apply this technique for the theoretical 


\section{Bashkirtseva et al.}

analysis of P-bifurcations of stochastic cycles of the Lorenz system. One of the main results of this analysis is a clarification of the probabilistic mechanism of D-bifurcation (stochastic chaotization) via P-bifurcation (first BSB).

\section{Backward Stochastic Bifurcations}

We will study P-bifurcations of stochastic cycles for parameter values $r_{1}, r_{2}, r_{3}$ introduced above.
Consider a deformation of the probabilistic distribution of random states in the stochastic 2-cycle for $r=r_{1}$ under increasing noise. A stochastically forced phase trajectory of system (1) leaves this deterministic 2-cycle [see Fig. 2(b)] and forms some stochastic bundle around it. For small noise, random trajectories are localized near the unforced cycle and form a stochastic 2-cycle [see Fig. 6(a)].

As the noise intensity increases, a dispersion of the stochastic bundle grows and noise-induced

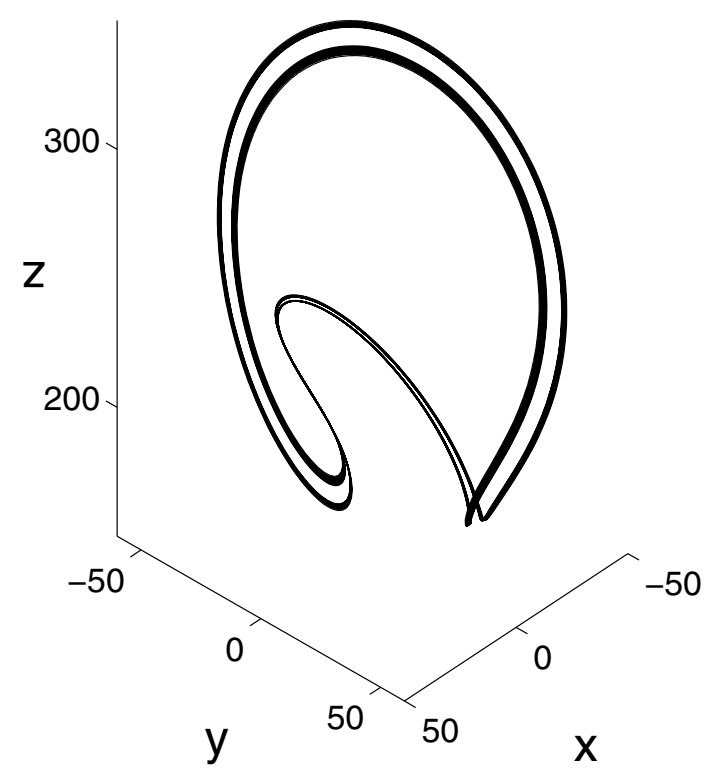

(a)

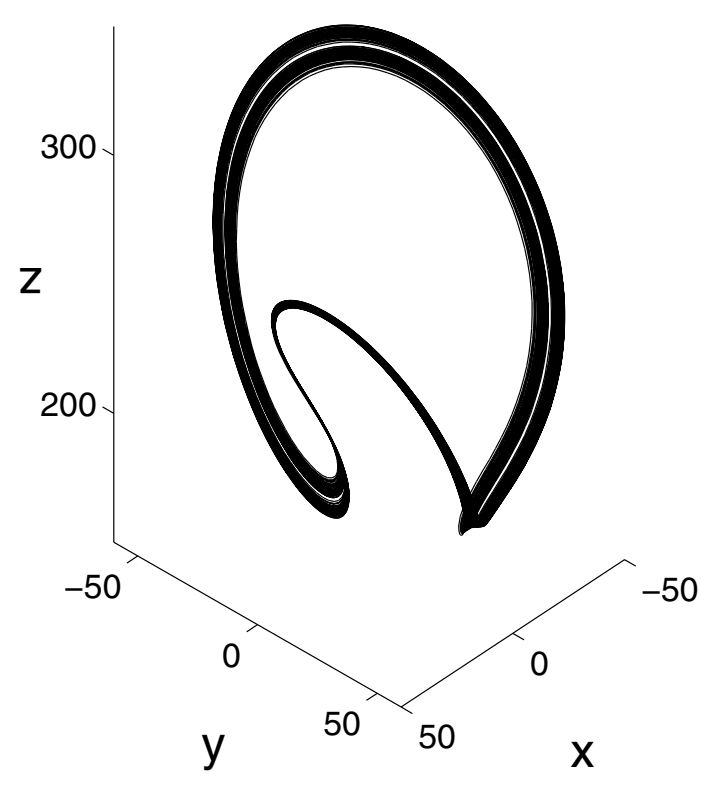

(b)

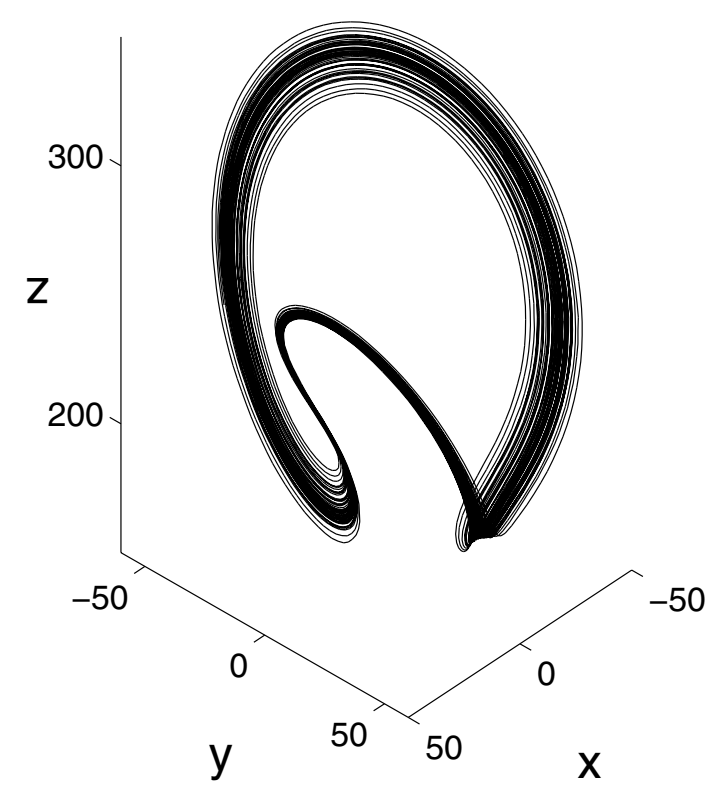

(c)

Fig. 6. Stochastic 2-cycles: (a) $\varepsilon=0.2$, (b) $\varepsilon=0.5$ and (c) $\varepsilon=1$. 
transitions of random trajectories between nearest loops of the 2-cycle are observed. Corresponding parts of the stochastic 2-cycle intersect each other [see Fig. 6(b)]. With a further growth of noise, the separate but close parts of the stochastic bundle are coalescing into a single one. The stochastically forced 2-cycle in Fig. 6(c) is similar to the stochastic 1-cycle in Fig. 3. This can be interpreted as a noise-induced transformation of the stochastic 2-cycle to the stochastic 1-cycle. Here, we observe a decreasing of the cycle multiplicity as noise intensity increases. We name this noise-induced phenomena [Bashkirtseva et al., 2010b] as backward stochastic bifurcation.

For any stochastic $2^{k}$-cycle, a cascade of the backward stochastic bifurcations can be observed: stochastic $2^{k}$-cycle $\rightarrow$ stochastic $2^{k-1}$-cycle $\rightarrow$ $\cdots \rightarrow$ stochastic 1-cycle. Such backward stochastic

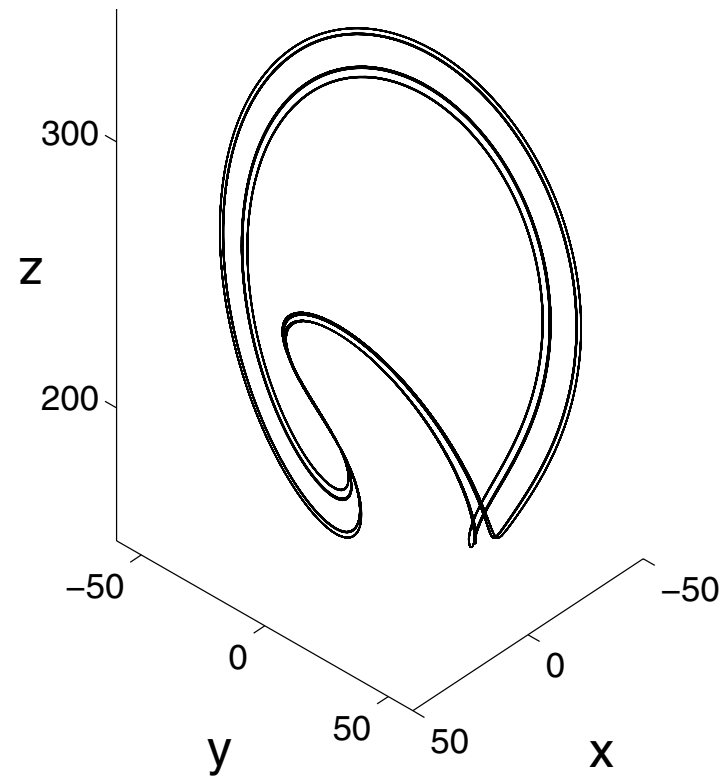

(a)

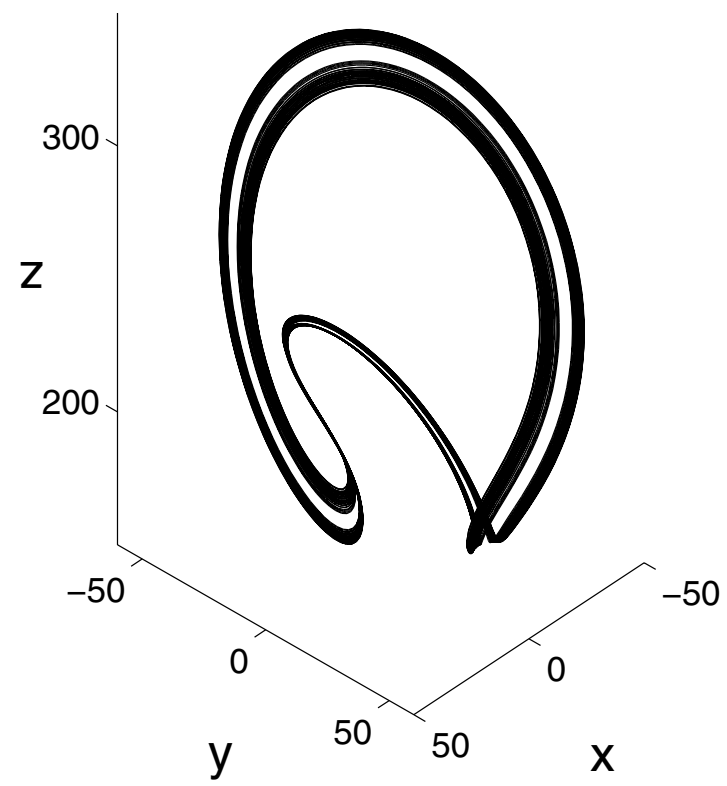

(b)

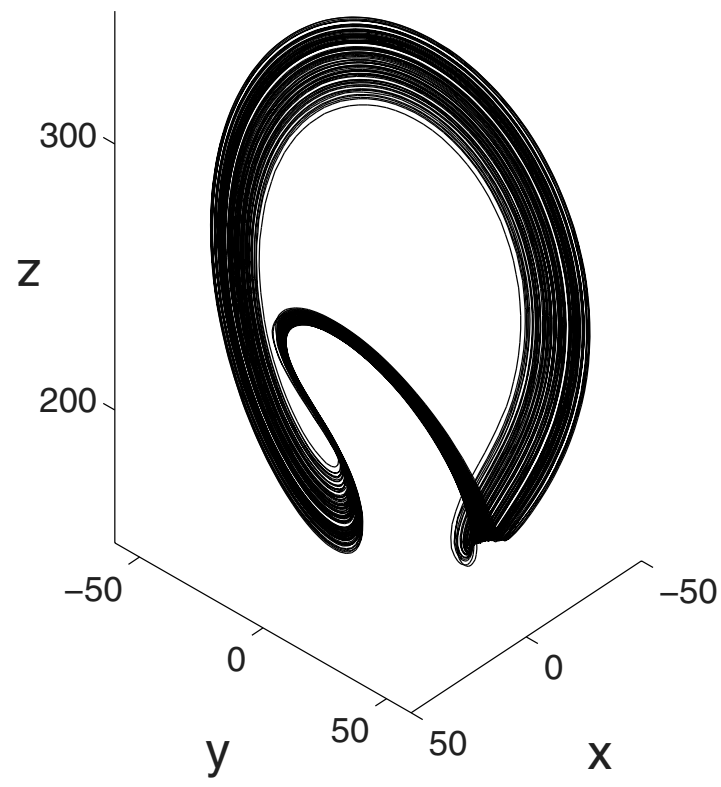

(c)

Fig. 7. Stochastic 4-cycles: (a) $\varepsilon=0.02$, (b) $\varepsilon=0.2$ and (c) $\varepsilon=1$. 


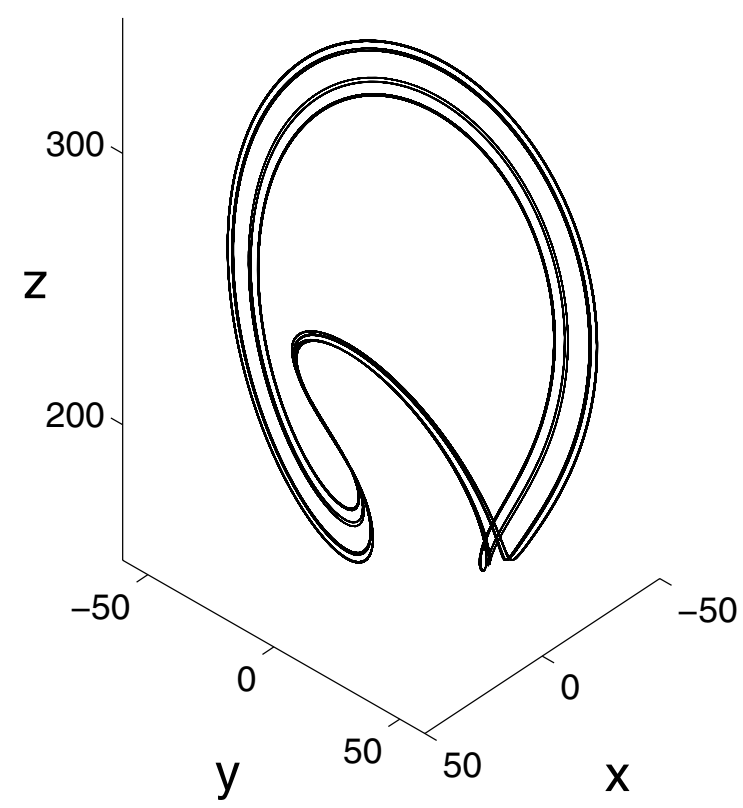

(a)

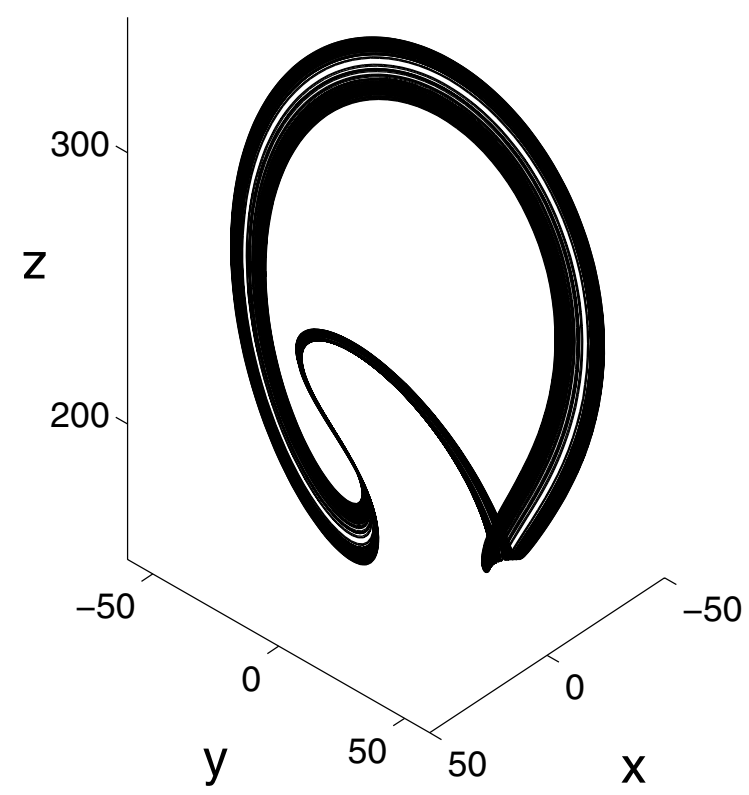

(c)

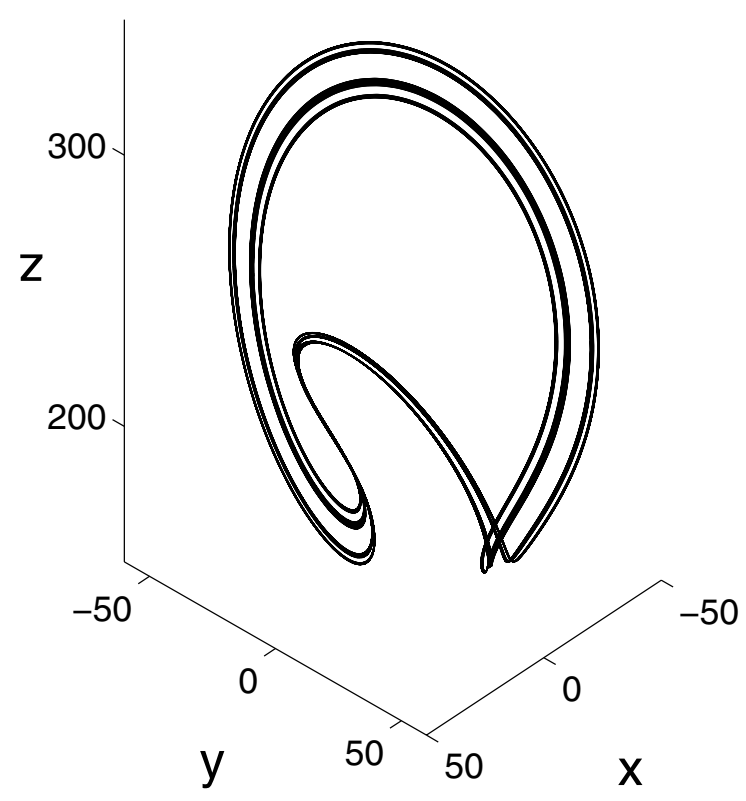

(b)

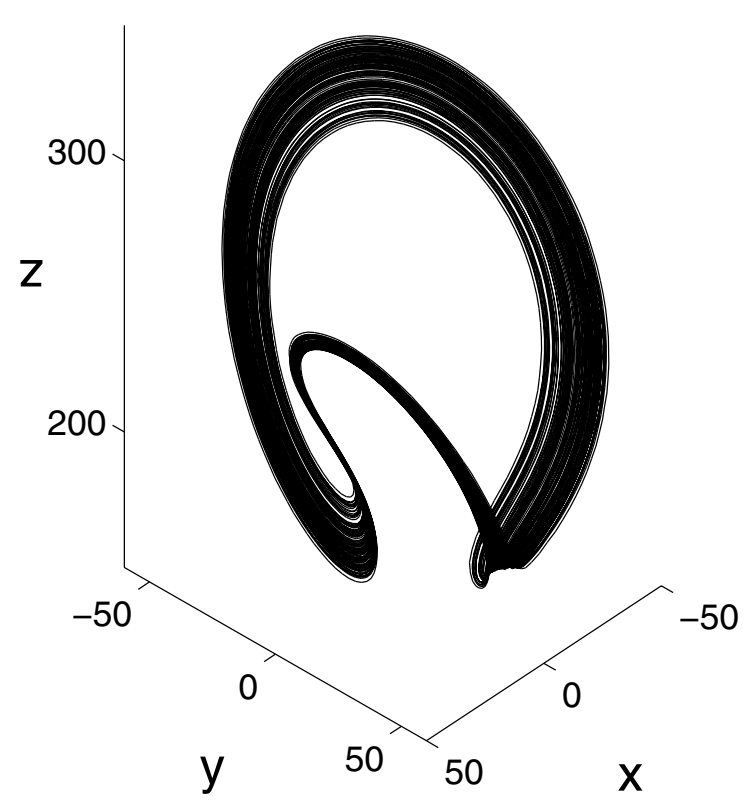

(d)

Fig. 8. Stochastic 8-cycles: (a) $\varepsilon=0.002$, (b) $\varepsilon=0.02$, (c) $\varepsilon=0.2$ and (d) $\varepsilon=1$.

bifurcations are demonstrated in Fig. 7 for 4-cycle with $r=r_{2}$ and Fig. 8 for 8-cycle with $r=r_{3}$. As one can see in Fig. 7(a) for $\varepsilon=0.02$, four loops of stochastic 4-cycle are definitely separated. For $\varepsilon=0.2$, the stochastic attractor looks like a stochastic 2-cycle [see Fig. 7(b)]. With further growth of $\varepsilon$, the stochastic attractor transforms into stochastic 1-cycle (see Fig. 7(c) for $\varepsilon=1$ ).
An analogous scenario of backward stochastic bifurcations is presented in Fig. 8 where the following cascade is observed: 8-cycle (Fig. 8(a) for $\varepsilon=0.002) \rightarrow$ 4-cycle (Fig. 8(b) for $\varepsilon=0.02) \rightarrow$ 2-cycle (Fig. 8(c) for $\varepsilon=0.2) \rightarrow 1$-cycle (Fig. 8(d) for $\varepsilon=1)$.

A formal mathematical study of this phenomenon of backward stochastic bifurcations 
(a)

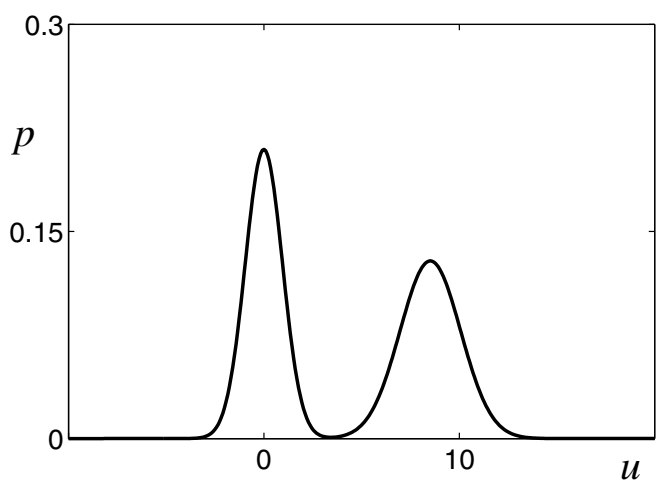

(b)

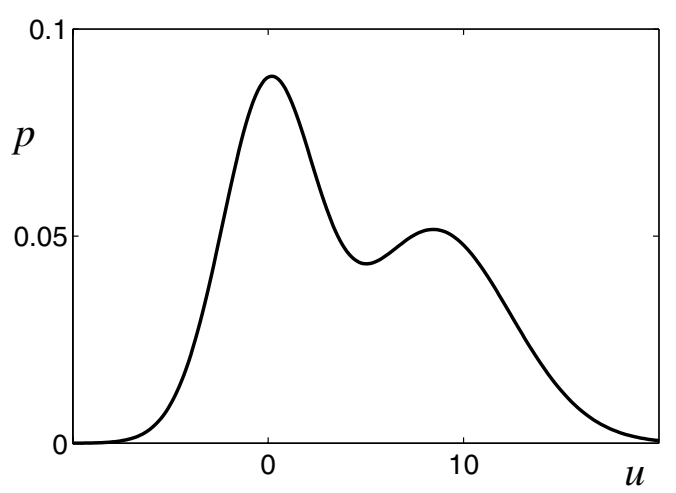

(c)

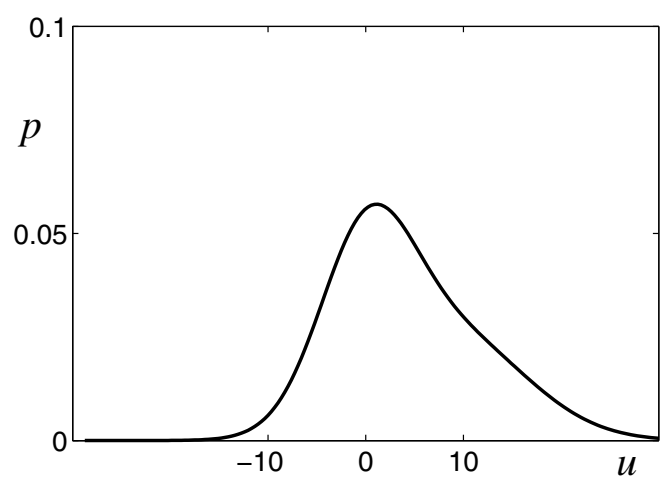

Fig. 9. Stochastic 2-cycles: transformation of pdf for $r_{1}=224.5$. (a) $\varepsilon=0.2$, (b) $\varepsilon=0.5$ and (c) $\varepsilon=1$.

(a)

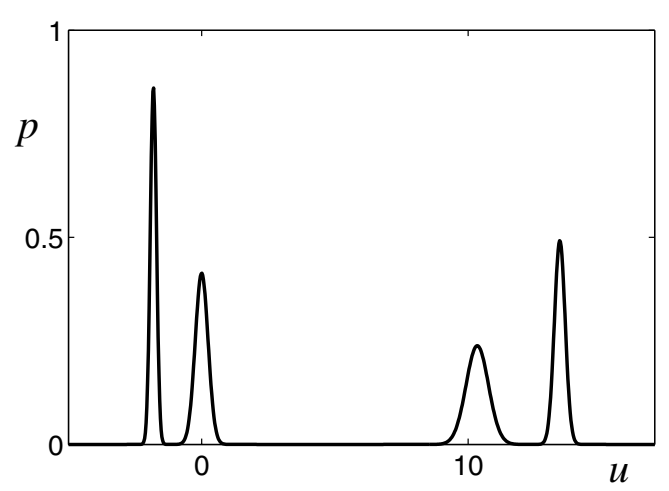

(b)

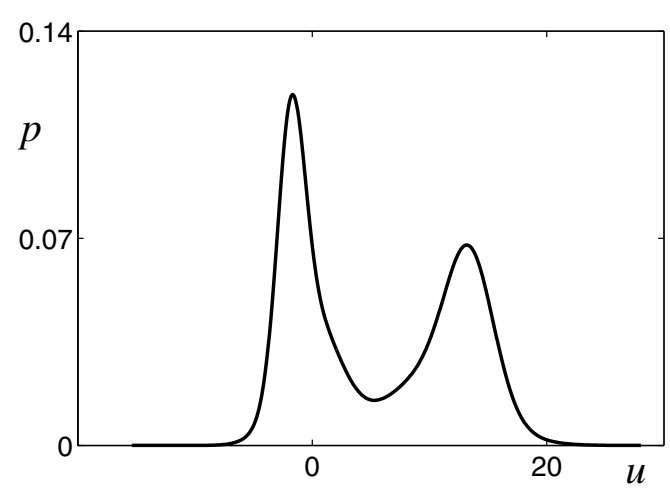

(c)

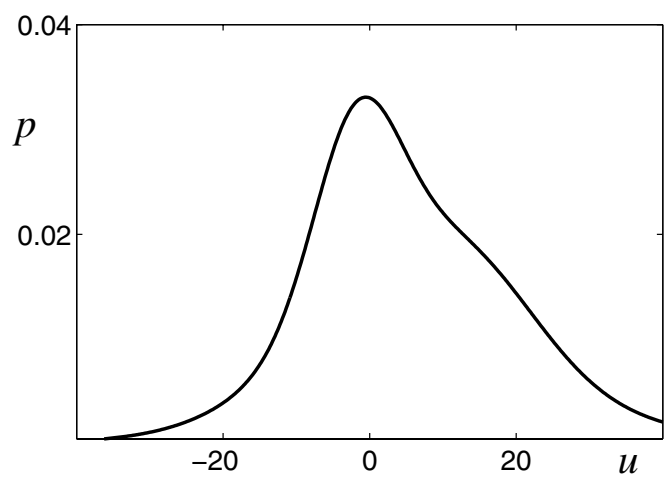

Fig. 10. Stochastic 4-cycles: transformation of pdf for $r_{2}=217.2$. (a) $\varepsilon=0.02$, (b) $\varepsilon=0.2$ and (c) $\varepsilon=1$. 


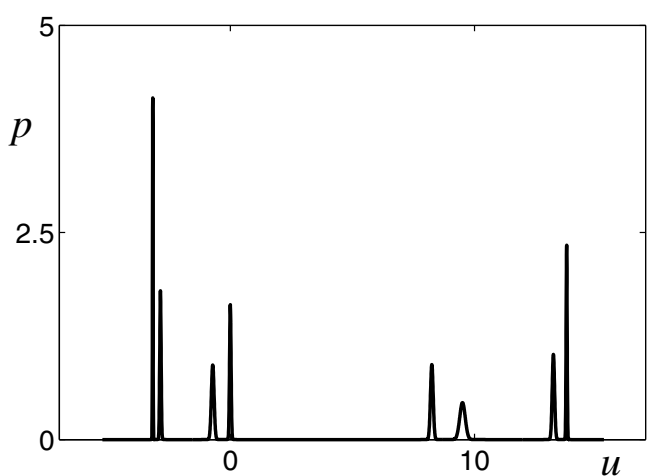

(a)

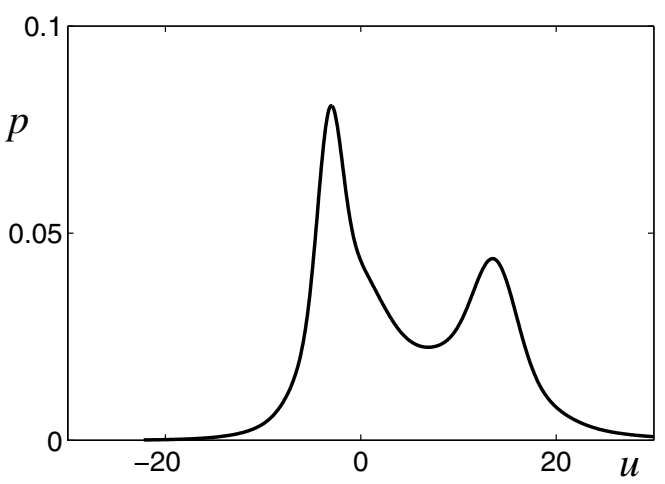

(c)

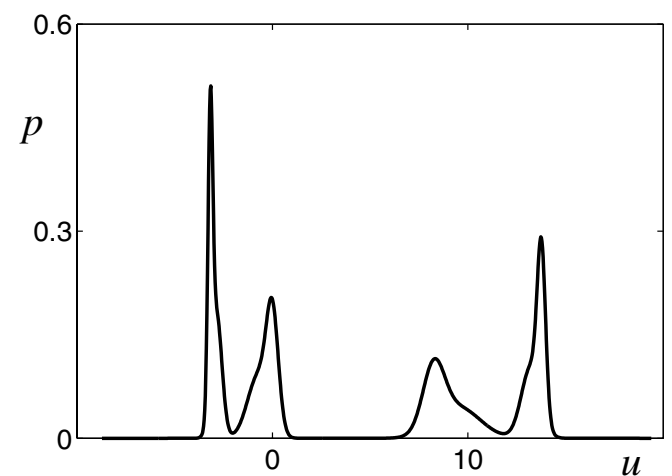

(b)

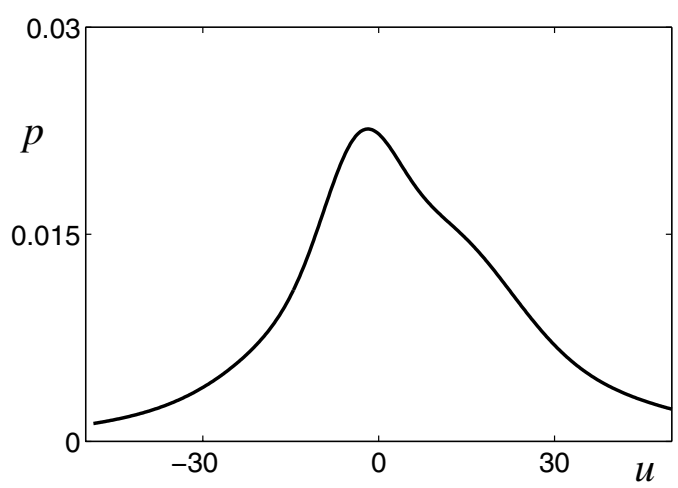

(d)

Fig. 11. Stochastic 8-cycles: transformation of pdf for $r_{3}=215.75$. (a) $\varepsilon=0.002$, (b) $\varepsilon=0.02$, (c) $\varepsilon=0.2$ and (d) $\varepsilon=1$.

presented here is carried out by the analysis of probability density functions. Each BSB is accompanied by a qualitative change of the probability density function. Here, for a detailed parametrical analysis of the 3D phenomenon of backward stochastic bifurcations, we use Poincaré sections approach which allows to reduce a dimension.

Fix a Poincaré section plane $\Pi$ and a line $l$ which belongs to $\Pi$. Let $u$ be a coordinate of the current point of this line $l$. Consider points of intersection of the stochastic attractor with $\Pi$. Denote by $p(u)$ a probability density function for projections of these intersection points onto the line $l$. Maxima of $p(u)$ mark zones of highest concentration of random states in the stochastic attractor. Now, we can reduce a study of BSB to the analysis of qualitative changes of $p(u, \varepsilon)$ curves. In this paper, for the function $p(u, \varepsilon)$, we will use a semianalytical approximation based on the stochastic sensitivity functions technique (see details in Appendix).

In Fig. 9, the plots of $p(u, \varepsilon)$ are presented for the same noise intensities $\varepsilon=0.2,0.5,1$ and $r=r_{1}$ as in Fig. 6. For small noise $(\varepsilon=0.2)$, a curve of the probability density function has two narrow peaks [Fig. 9(a)]. The width of these peaks is rising as $\varepsilon$ increases. For further noise increases, a process of peaks coalescing is observed [see Fig. 9(b)]. As one can see, a local minimum of $p(u)$ increases but the general graphic shape remains bimodal and a number of local extreme points equals three. For some threshold value $\varepsilon^{*}$, a qualitative change of $p(u)$ shape occurs. A form of this curve becomes unimodal [see Fig. 9(c)]. Two separate peaks transform into one and the only maximum remains. Further increasing of $\varepsilon$ results only in the extension of the single peak width. We can use this transformation of $p(u, \varepsilon)$ from bimodal to unimodal form as a formal indicator of BSB.

For cycles of higher multiplicity, a chain of successive backward stochastic bifurcations is observed. Each BSB is accompanied by the change of the quantity of pdf maxima. Consider in detail the transformations of pdf for 4-cycle with $r=r_{2}$ (see Fig. 10) and for 8-cycle with $r=r_{3}$ (see Fig. 11). 
As one can see in Fig. 10, for small noise with $\varepsilon=0.02$, four sharp peaks of pdf confirm that the stochastic attractor in Fig. 7(a) is a stochastic 4-cycle. For $\varepsilon=0.2$, the pdf transforms into the bimodal form, and the stochastic attractor in Fig. 7(b) is a stochastic 2-cycle. Here, the first BSB $4 \rightarrow 2$ is observed.

With further growth of $\varepsilon$, a shape of pdf curve becomes unimodal and the stochastic attractor (see Fig. 7(c) for $\varepsilon=1$ ) is transformed into the stochastic 1-cycle (second BSB $2 \rightarrow 1$ ). Values of $\varepsilon$ which correspond to changes of the modality of pdf curve, allow to estimate the points of successive backward stochastic bifurcations: $4 \rightarrow 2,2 \rightarrow 1$.

A chain of successive BSB $8 \rightarrow 4 \rightarrow 2 \rightarrow 1$ for the stochastically forced 8-cycle with $r=r_{3}$ is presented in Figs. 11(a)-11(d).

Details of qualitative changes of pdf for the increasing noise intensity are shown in Fig. 12. Here, the maxima (solid lines) and minima (dashed lines) are plotted. Each BSB is accompanied by the annihilation of maxima through the confluence with corresponding minima.

An overview of results of our analysis is assembled in Table 1. Here, for cycles of the various multiplicity, the threshold values of the noise intensity which correspond to backward stochastic bifurcations are presented. It is interesting to compare these results concerning P-bifurcations with our analysis of D-bifurcations presented in Sec. 2. Threshold noise intensity $\varepsilon_{1}^{*}=0.61$ which marks BSB $2 \rightarrow 1$ for $r_{1}$ corresponds to the onset of

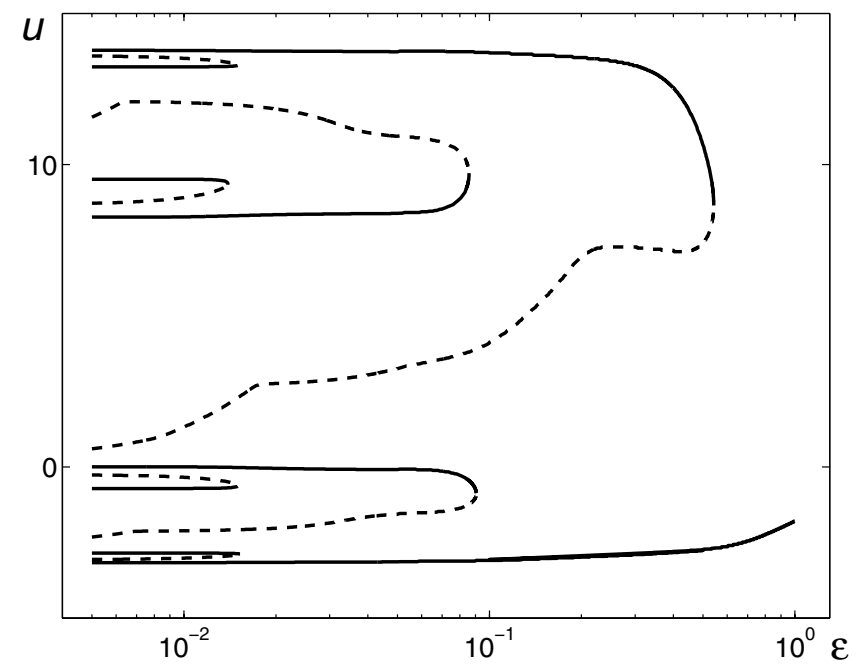

Fig. 12. Maxima (solid lines) and minima (dashed lines) of pdf for $r_{3}=215.75$.
Table 1. Threshold noise intensity values of BSB.

\begin{tabular}{lcccc}
\hline$r$ & $16 \rightarrow 8$ & $8 \rightarrow 4$ & $4 \rightarrow 2$ & $2 \rightarrow 1$ \\
\hline$r_{1}$ & - & - & - & 0.61 \\
$r_{2}$ & - & - & 0.094 & 0.68 \\
$r_{3}$ & - & 0.015 & 0.086 & 0.60 \\
$r_{4}$ & 0.0024 & 0.013 & 0.071 & 0.44 \\
\hline
\end{tabular}

chaotization of stochastic 2-cycle (see Fig. 5). This fact can be explained by the following. In phase space, loops of the stable deterministic 2-cycle are surrounded by the basins of attraction divided by separatrix. Near the separatrix, a dynamical system is locally unstable and a divergence of phase trajectories is observed. For small noise, random trajectories are concentrated in basins of attraction and the corresponding stochastic attractor is regular. As noise intensity increases and BSB occurs, the random trajectories cross the separatrix and with a high probability fall within zones of divergence. As a result, the dynamics of the perturbed system as a whole becomes chaotic and the largest Lyapunov exponent becomes positive. Thus, BSB $2 \rightarrow 1$ implies a chaotization of stochastic attractor.

An analogous probabilistic mechanism takes place for cycles of higher multiplicity too. Indeed, for example, the threshold noise intensity $\varepsilon_{2}^{*}=$ 0.094 (see Table 1) which marks the first BSB $4 \rightarrow 2$ for $r_{2}$ corresponds to the onset of chaotization of stochastic 4-cycle (see Fig. 5).

\section{Conclusion}

We have studied the phenomena of noise-induced chaotization and backward stochastic bifurcations of randomly forced multiple limit cycles of the Lorenz model in a period-doubling bifurcation zone. Our investigation is based on the analysis of Lyapunov exponents and the stochastic sensitivity function technique. For stochastic cycles of various multiplicity, the threshold values of noise intensity corresponding to both transitions from order to chaos and backward stochastic bifurcations are found. It was shown that for a limit cycle with multiplicity two and higher, the threshold value of the noise intensity which marks the onset of chaos agrees with first BSB. Due to the universality of the presented method, it can be applied for the analysis of $\mathrm{P}$ - and D-bifurcations for any 3D-model with a period-doubling bifurcation zone. 


\section{Acknowledgments}

This work was partially supported by the Ministry of Education and Science of Russia (projects 1.1099.2011, 14.A18.21.0364) and RFBR (13-0800069).

\section{References}

Anishchenko, V. S., Khovanov, I. A., Khovanova, N. A., Luchinsky, D. G. \& McClintock, P. V. E. [2001] "Noise-induced escape from the Lorenz attractor," Fluct. Noise Lett. 1, 27-34.

Anishchenko, V. S., Astakhov, V. V., Neiman, A. B., Vadivasova, T. E. \& Schimansky-Geier, L. [2007] Nonlinear Dynamics of Chaotic and Stochastic Systems. Tutorial and Modern Development (Springer-Verlag, Berlin/Heidelberg).

Arnold, L., Sri Namachchivaya, N. \& Schenk-Hoppe, K. [1996] "Toward an understanding of stochastic Hopf bifurcation: A case study," Int. J. Bifurcation and Chaos 6, 1947-1975.

Arnold, L. [1998] Random Dynamical Systems (SpringerVerlag).

Arnold, L., Bleckert, G. \& Schenk-Hoppe, K. [1999] "The stochastic Brusselator: Parametric noise destroys Hopf bifurcation," Stochastic Dynamics (Springer, NY), pp. 71-92.

Bashkirtseva, I. A. \& Ryashko, L. B. [2004] "Stochastic sensitivity of 3D-cycles," Math. Comput. Simul. 66, $55-67$.

Bashkirtseva, I. A. \& Ryashko, L. B. [2009] "Constructive analysis of noise-induced transitions for coexisting periodic attractors of Lorenz model," Phys. Rev. E 79, 041106.

Bashkirtseva, I. A., Ryashko, L. B. \& Schurz, H. [2009] "Analysis of noise-induced transitions for Hopf system with additive and multiplicative random disturbances," Chaos Solit. Fract. 39, 72-82.

Bashkirtseva, I., Chen, G. \& Ryashko, L. [2010a] "Analysis of stochastic cycles in the Chen system," Int. J. Bifurcation and Chaos 20, 1439-1450.

Bashkirtseva, I. A., Ryashko, L. B. \& Stikhin, P. [2010b] "Noise-induced backward bifurcations of stochastic 3D-cycles," Fluct. Noise Lett. 9, 89-106.

Bashkirtseva, I., Chen, G. \& Ryashko, L. [2012] "Analysis of noise-induced transitions from regular to chaotic oscillations in the Chen system," Chaos 22, 033104 .

Crutchfield, J. P., Nauenberg, M. \& Rudnick, J. [1981] "Scaling for external noise at the onset of chaos," Phys. Rev. Lett. 46, 933-935.

Crutchfield, J. P., Farmer, J. \& Huberman, B. A. [1982] "Fluctuations and simple chaotic dynamics," Phys. Rep. 92, 45-82.
Dorfle, M. \& Graham, R. [1983] "Probabilty density of the Lorenz model," Phys. Rev. A 27, 1096.

Fedchenia, I. I., Mannella, R., McClintock, P. V. E., Stein, N. D. \& Stocks, N. G. [1992] "Influence of noise on periodic attractors in the Lorenz model: Zerofrequency spectral peaks and chaos," Phys. Rev. A 46, 1769-1774.

Freidlin, M. I. \& Wentzell, A. D. [1984] Random Perturbations of Dynamical Systems (Springer, NY).

Gao, J. B., Tung, W. \& Rao, N. [2002] "Noiseinduced Hopf-bifurcation-type sequence and transition to chaos in the Lorenz equations," Phys. Rev. Lett. 89, 254101.

Horsthemke, W. \& Lefever, R. [1984] Noise-Induced Transitions (Springer, Berlin).

Huang, Z., Yang, Q.-G. \& Cao, J. [2011] "Stochastic stability and bifurcation analysis on Hopfield neural networks with noise," Expert Syst. Appl.: An Int. J. 38, 10437-10445.

Ito, H. M. [1984] "Ergodicity of randomly perturbed Lorenz model," J. Stat. Phys. 35, 151-159.

Keizer, J., Fox, R. F. \& Wagner, J. [1993] "On the amplification of molecular fluctuations for nonstationary systems: Hydrodynamical fluctuations for the Lorenz model," Phys. Lett. A 175, 17-22.

Lai, Y.-C., Liu, Z., Billings, L. \& Schwartz, I. B. [2003] "Noise-induced unstable dimension variability and transition to chaos in random dynamical systems," Phys. Rev. E 67, 026210.

Landa, P. S. \& McClintock, P. V. E. [2000] "Changes in the dynamical behavior of nonlinear systems induced by noise," Phys. Rep. 323, 1-80.

Liu, Z., Lai, Y.-C., Billings, L. \& Schwartz, I. B. [2002] "Transition to chaos in continuous-time random dynamical systems," Phys. Rev. Lett. 88, 124101.

Liu, Z. \& Ma, W. [2005] "Noise induced destruction of zero Lyapunov exponent in coupled chaotic systems," Phys. Lett. A 343, 300-305.

Mayer-Kress, G. \& Haken, H. [1981] "The influence of noise on the logistic model," J. Stat. Phys. 29, 149171.

Mil'shtein, G. N. \& Ryashko, L. B. [1995] "A first approximation of the quasipotential in problems of the stability of systems with random nondegenerate perturbations," J. Appl. Math. Mech. 59, $47-56$.

Pradas, M., Tseluiko, D., Kalliadasis, S., Papageorgiou, D. T. \& Pavliotis, G. A. [2011] "Noise induced state transitions, intermittency and universality in the noisy Kuramoto-Sivashinksy equation," Phys. Rev. Lett. 106, 060602.

Rasmussen, M. [2007] Attractivity and Bifurcation for Nonautonomous Dynamical Systems, Lecture Notes in Mathematics, Vol. 1907 (Springer). 
Ryagin, M. \& Ryashko, L. [2004] "The analysis of the stochastically forced periodic attractors for Chua's circuit," Int. J. Bifurcation and Chaos 14, 39813987.

Ryashko, L., Bashkirtseva, I., Gubkin, A. \& Stikhin, P. [2009] "Confidence tori in the analysis of stochastic 3D-cycles," Math. Comput. Simul. 80, 256-269.

Sparrow, C. [1982] The Lorenz Equations: Bifurcations, Chaos, and Strange Attractors (Springer-Verlag, NY).

Tel, T. \& Lai, J.-C. [2010] "Quasipotential approach to critical scaling in noise-induced chaos," Phys. Rev. E 81, 056208.

Tung, W.-W., Hu, J., Gao, J. \& Billock, V. A. [2008] "Diffusion, intermittency, and noise-sustained metastable chaos in the Lorenz equations: Effects of noise on multistability," Int. J. Bifurcation and Chaos 18, 1749-1758.

\section{Appendix. Stochastic Sensitivity Functions Technique}

Consider a general system of stochastic differential equations

$$
\dot{x}=f(x)+\varepsilon \sigma(x) \dot{w},
$$

where $x$ is an $n$-vector, $f(x)$ is a $n$-vector function, $w(t)$ is a $n$-dimensional standard Wiener process, $\sigma(x)$ is a $n \times n$-matrix-valued function of the disturbances with intensity $\varepsilon$. Suppose that the corresponding deterministic system (A.1) $(\varepsilon=0)$ has a $T$-periodic solution $x=\xi(t)$ with an exponentially stable phase curve $\gamma$ (limit cycle).

Trajectories of the randomly forced system (A.1) leave the deterministic attractor and form a corresponding stochastic attractor with stationary probabilistic distribution $\rho(x, \varepsilon)$. The stationary Kolmogorov-Fokker-Planck equation provides a detailed probabilistic description of the random distribution $\rho(x, \varepsilon)$. In general, the analysis of this equation appears to be technically a very difficult problem. Under these circumstances, an approximation

$$
\rho(x, \varepsilon) \approx K \cdot \exp \left(-\frac{v(x)}{\varepsilon^{2}}\right)
$$

based on the quasipotential $v(x)$ is used [Freidlin \& Wentzell, 1984] for low noise.

Let $\Pi_{t}$ be a hyperplane orthogonal to the cycle at the point $\xi(t)(0 \leq t<T)$. In this case, for the Poincaré section $\Pi_{t}$ in the neighborhood of the point $\xi(t)$, using a quadratic approximation of the quasipotential [Mil'shtein \& Ryashko, 1995], one can write [Bashkirtseva \& Ryashko, 2004] Gaussian approximation for the probability density

$$
\rho_{t}(x, \varepsilon) \approx K \exp \left(-\frac{(x-\xi(t)), W^{+}(t)(x-\xi(t))}{2 \varepsilon^{2}}\right)
$$

with the covariance matrix $\varepsilon^{2} W(t)$. Here the matrix function $W(t)$ is singular, the sign " + " means a pseudoinversion. The matrix $W(t)$ characterizes the dispersion of the points of intersection of the random trajectories with $\Pi_{t}$. The function $W(t)$ is a stochastic sensitivity function (SSF) of the limit cycle. This function allows to describe the most essential geometrical features of the probabilistic distribution of the random trajectories around the deterministic cycle. Such description can be provided on the basis of the confidence tori technique [Ryashko et al., 2009].

The matrix $W(t)$ is a unique solution [Bashkirtseva \& Ryashko, 2004] of the Lyapunov equation

$$
\dot{W}=F(t) W+W F^{\top}(t)+P(t) S(t) P(t)
$$

with conditions

$$
W(0)=W(T), \quad W(t) r(t) \equiv 0,
$$

where $F(t)=\frac{\partial f}{\partial x}(\xi(t)), S(t)=\sigma(\xi(t)) \sigma^{\top}(\xi(t))$, $r(t)=f(\xi(t)), P(t)$ is a matrix of the orthogonal projection onto the hyperplane $\Pi_{t}$.

Consider now an arbitrary Poincaré section plane $\Pi$ with unit directing vector $b$, and a line $l$ which belongs to $\Pi$. On the line $l$, define a coordinate system with the origin point $\eta$ and a unit directing vector $a$. Then any point $x \in l$ has a corresponding scalar coordinate $u: x(u)=\eta+u a$.

Let $\xi_{i}=\xi\left(t_{i}\right)(i=1, \ldots, k)$ be points of the intersection of the deterministic cycle $\gamma$ with $\Pi$. For any point $\xi_{i}$, we find SSF matrix $W_{i}=W\left(t_{i}\right)$. A corresponding SSF matrix $\Phi_{i}$ for the Poincaré section $\Pi$ can be calculated as

$$
\Phi_{i}=B_{i} W_{i} B_{i}^{\top}, \quad B_{i}=\frac{I-r_{i} b^{\top}}{r_{i}^{\top} b}, \quad r_{i}=\frac{f\left(\xi_{i}\right)}{\left\|f\left(\xi_{i}\right)\right\|} .
$$

The value $\sigma_{i}^{2}=a^{\top} \Phi_{i} a$ is a quantity of the stochastic sensitivity for the cycle at the point $\xi_{i}$ in a direction of the vector $a$.

Let $u_{i}$ be coordinates of the projections of the points $\xi_{i}$ onto $l$. Consider points of intersection 
of the stochastic cycle with $\Pi$. Denote by $p(u, \varepsilon)$ a probability density function for projections of these intersection points onto the line $l$. Values $u_{i}$ and $\sigma_{i}(i=1, \ldots, k)$ allow us to find a Gaussian approximation for this probability density function as follows

$$
p(u, \varepsilon)=\frac{1}{k} \sum_{i=1}^{k} \frac{1}{\sqrt{2 \pi} \varepsilon \sigma_{i}} \exp \left(-\frac{\left(u-u_{i}\right)^{2}}{2 \varepsilon^{2} \sigma_{i}^{2}}\right) .
$$


Copyright of International Journal of Bifurcation \& Chaos in Applied Sciences \& Engineering is the property of World Scientific Publishing Company and its content may not be copied or emailed to multiple sites or posted to a listserv without the copyright holder's express written permission. However, users may print, download, or email articles for individual use. 